\title{
La construcción social y simbólica de las Chavas Activas Punks
}

Inés Cornejo Portugal ${ }^{1}$

\section{Introducción: El "mundo de la vida" de las CHAP}

\begin{abstract}
C.
CÓMO ACCEDER de manera analítica al espacio de delimitación identitaria del colectivo Chavas Activas Punks (CHAP)? ¿De qué forma podemos estudiar el "nosotras" de estas mujeres punketas? ¿.Cómo entender, desde su producción cultural simbólica, la reafirmación de un colectivo diferente a la banda masculina?

Para aproximarse a las CHAP y a sus prácticas culturales, es pertinente acercarse desde un enfoque microscópico a las maneras cotidianas, rutinarias e intersubjetivas desde las cuales se construye el ser chava banda, puesto que en anteriores prospecciones se realizaron acercamientos más bien macroscópicos, considerando el análisis de la cultura y de las instituciones sociales (Urteaga y Cornejo, 1996-1998).

Así, para explotar el "mundo de la vida" de estas jóvenes punketas, es conveniente estudiar cómo ellas construyen experiencialmente su mundo subjetivo e intersubjetivo, tanto en el ámbito familiar inmediato como en la banda intergenérica (mixta) y en el colectivo de puras chavas, las CHAP.

Tipificaciones, recetas, acervo social de conocimiento común, rol distanciamiento de rol e identidad, son algunas de las categorías que iluminan el diálogo establecido entre la propuesta teórica fenomenoiogici .. llfred Schutz y la información empírica claborada.

Con el propósito de analizar los espacios simbólicos de las relaciones significativas de las protagonistas, en este caso, las CHAP, se definieron las siguientes vías:
\end{abstract}

1. Universidad Iberoamericana, Plantel Santa Fe. 
1. Explotar e interpretar los significados que tienen para las jóvenes involucradas el hecho de ser día con día chava activa punk.

2. Analizar el escenario banda (intergenérico e intragenérico) como lugar simbólico de la relación "nosotros".

3. Estudiar los procesos de reconocimiento y autodefinición femenina que las CHAP generan al impulsar y forjar una organicidad propia al interior de la banda intergenérica.

Las dos primeras vías, el significado del mundo cotidiano y el escenario de la relación "nosotros", posibilitan estudiar la manera en la cual las CHAP redefinen su mundo familiar cotidiano y lo desplazan hacia la banda masculina. Al interior de ésta se apropian de un espacio, lo que a la vez es creador de identidad y edificador de autoafirmación positiva de su ser mujer.

\section{Antecedentes}

En 1996 nos propusimos desarrollar una investigación sobre la construcción identitaria de algunas de las chavas pertenecientes al Colectivo Chavas Activas Punks. Para iniciar el trabajo, definimos la propuesta teórica-metodológica del mismo y decidimos realizar entrevistas a las líderes fundadoras del Colectivo, puesto que fueron ellas quienes gestaron la propuesta general del proyecto y elaboraron las ideas fuertes del movimiento desde que éste fue creado en octubre de 1987.

El colectivo CHAP estuvo integrado por más de treinta mujeres que vivían en la ciudad de México y en ciudad Nezahualcóyotl. Luego de participar en bandas intergenéricas (mixtas), ellas decidieron congregarse en una banda intragenérica (femenina) con el propósito de crear nuevas formas de organización. A través de manifestaciones culturales, tales como fanzines, performances, poesías o conciertos, se propusieron demostrar a los hombres que eran "iguales" a ellos en inteligencia y capacidad organizativa, pero "diferentes", pues eran mujeres y tenían un mundo interior distinto. En los primeros años de la década de los noventa algunas de ellas desertaron del colectivo, mientras que otras mantuvieron iniciativas dentro de su nuevo rol de madres.

En este sentido, formulamos dos hipótesis de partida. La primera exploraba cómo los contextos familiares, escolares y vanidosos tenían que ver con la conformación de estas mujeres como chavas banda. Es decir, nos preguntamos por aquellas razones que hacen que una joven clasemediera o clasemediera baja busque en el espacio de la banda un continente afectivo emocional y de "orden" frente al aparente caos de su vida familiar y cotidiana. La segunda hipótesis daba cuenta de cómo la pertenencia al espacio bandoso intergenérico de alguna 
manera forjaba ciertos elementos de su identidad femenina y, además, de su ser chava "activa" y propositiva.

Nos propusimos realizar entrevistas en profundidad, las cuales, durante el desarrollo de las mismas, se fueron trastocando en relatos de vida por las características que iban asumiendo. Es decir, las chavas hablaban en primera persona y daban cuenta de los diferentes acontecimientos desde sus propias perspectivas y desde su mundo subjetivo/afectivo. Por medio de este instrumento se trató de aprehender las experiencias de estas jóvenes y en cómo le daban sentido y explicaban dichas experiencias.

Para realizar las entrevistas, claboramos una guía abierta, flexible, no directiva, siguiendo la propuesta de S. J. Taylor y R. Bodgan. Esos autores definen la entrevista en profundidad como "encuentros reiterados cara a cara entre el investigador y los informantes, encuentros dirigidos hacia la comprensión de sus vidas, experiencias o situaciones, tal como las expresan con sus propias palabras" (Taylor y Bodgan, 1992:101). La guía contenía aquellas áreas generales que darían cuenta de las hipótesis de partida.

Asimismo, el proceso de la entrevista fue desarrollado en un ambiente de mutua confianza entre las coproductoras del relato. Dicha confianza es el resultado de varios años de trabajo realizado anteriormente con motivo de la elaboración de la tesis de maestría de una de las autoras de la investigación mayor.

\section{De los conceptos a la interpretación: Mundo cotidiano de las CHAP: familia y banda}

De acuerdo con Schutz, el "mundo cotidiano" es aquel espacio donde los sujetos usan tipificaciones y recetas para desarrollarse y relacionarse. En él, las personas participan con una "actitud natural", es decir, sin cuestionar su realidad (Ritzer: 271). El mundo de la vida es intersubjetivo, pre-existente y, además, escenario y objeto de las acciones e interacciones que permiten tanto identificar al grupo de referencia como distinguir entre un "nosotros" y un "ellos".

En el mundo cotidiano, las acciones de los sujetos vienen determinadas por las experiencias anteriores que las personas adquieren y almacenan a través del proceso de socialización. Esta relación experiencial reactualiza las tipificaciones preconstituidas derivadas de la propia sociedad y del acervo social de conocimiento. Mientras las tipificaciones se refieren a la realización que se establece con las personas, las recetas permiten organizar y controlar las situaciones cotidianas en las que se participa. Cuando las recetas habituales no responden a situaciones problemáticas o conflictivas, se crean fórmulas innovadoras - nuevas recetas- que son incorporadas al acervo social. 
En principio, el mundo cotidiano de estas jóvenes recoge la relación primaria socioafectiva familiar y, de manera especial, la ausencia o "falta de carácter" del padre, quien aparece como débil, pasivo, dominado y sin capacidad propositiva. Además se da una relación ambivalente con las madres, fuertes, agresivas y activas (en términos de proveedoras), pero "incapacitadas" de expresar y dar afecto.

En los hogares de esas CHAP se percibe la presencia de mujeres fuertes (madres, abuelas, tías) frente a hombres frágiles (padres y hermanos), "valemadristas", no comprometidos ni con los hijos ni con la familia. Dicha debilidad se expresa en su nula intervención en la esfera doméstica copada por las figuras femeninas internas o sustitutas (abuelas, tías).

En ese contexto las chavas parecen modificar sus "recetas de acción comprobada", para manejar y organizar una situación familiar aparentemente conflictiva. Es probable que las afirmaciones contradictorias respecto de la madre indiquen una búsqueda de una forma distinta de ser con ella; es decir, comprometida pero también afectiva y preocupada por los otros.

En segundo lugar, el mundo cotidiano de las CHAP se conforma a partir de su interacción con los padres, hombres y mujeres, en este espacio predominantemente masculino, el de las bandas punks. En éste, las chavas resuelven, en un primer momento, sus demandas de afecto, se sienten requeridas y aceptadas por los varones.

Sin embargo, el descubrimiento de sí mismas, como mujeres, ocurre a través del "deseo de posesión" del otro masculino hacia ellas. En la relación con los hombres, las chavas van en búsqueda de estima y reconocimiento, pero en otro masculino les responde desde su acervo social que conduce su acción más o menos habitual, es decir, reclama de la chava, aún al interior de la banda, un comportamiento aceptado y reconocido como "femenino"; ser pareja de algún chavo, ser enfermera o cocinera, en síntesis, ser proveedora y, de alguna manera, protectora - se trate de hijos, amantes o maridos- de los miembros masculinos de la banda.

En contraparte, las chavas negocian y elaboran nuevas recetas y tipificaciones distanciadas de su acervo social de origen, para autoafirmar positivamente su ser mujer y generar la construcción de un espacio propio al interior de la banda masculina. 


\section{La banda: mediación sustantiva para la construcción de la identidad femenina}

La "relación-nosotros" es, para Schutz, el escenario que se construye a través de las relaciones cara-a-cara, en la cual los co-partícipes son mutuamente conscientes de ellos mismos y participan solidariamente en la vida de cada uno durante algún tiempo, por corto que sea (Schutz, 1932/1969: 164, en Ritzer: 275).

Para las CHAP, el espacio banda es el lugar simbólico donde se concentran con mayor fuerza las interacciones significativas. Estas interacciones se elaboran tanto en las relaciones cara-a-cara intragenéricas hacia afuera con los pares y, también, con los chavos y chavas de las otras bandas.

En el colectivo femenino, las chavas desarrollan un intenso nivel de identidad, están familiarizadas unas con otras, conocen sus historias y trayectorias personales. Además, varias de ellas comparten experiencias familiares comunes: son amigas desde la escuela, frecuentan el mismo barrio y asisten casi siempre a los mismos sitios para divertirse, estar bien o rolarla con la banda.

Ante la ausencia o incapacidad de los padres, las madres de las CHAP son quienes ponen los límites a las hijas, las mandan a trabajar, deciden lo que deben hacer y las obligan a obtener algún ingreso para colaborar con la manutención del hogar. Estos hechos generan enfrentamientos entre madres e hijas, que para algunas de las CHAP se "resuelven" con su "refugio" en la banda. "Para mí, mi familia es la banda", es una afirmación recurrente en el discurso de las chavas. Frente al caos familiar en el que la mayoría se encuentra, el lugar bandoso parece convertirse paradójicamente en un "espacio de orden" en el cual su vida cobra sentido: “...empezamos a jalar un buen de banda, al estar juntas funcionaba. Sentíamos la vibra que nos daba fuerza, la unión nos hacía sentir chingonas: nadie nos podía destruir".

Estas frases sintetizan el surgimiento del Colectivo Chavas Activas Punks a finales de 1987. El activismo en el que "se clavan" las reúne la mayor parte del día, estrechan lazos de confianza actuando casi como hermanas. Esta inmediatez cotidiana las acerca personalmente y les permite "penetrar en el conocimiento del otro".

Lo anterior generó conflictos con los chavos, quienes, al verse excluidos de1 grupo, empezaron a tildarlas de "putas", "lesbianas" que "se consolaban entre ellas", "antihombres" y "marimachos". Las marginaron por algún tiempo, les retiraron la palabra, boicotearon sus acciones (no compraban el fanzine que ellas editaban, entraban de "a portazo" en las tocadas que organizaban) e, incluso, agredieron físicamente a las líderes.

De manera contradictoria, la banda es hacia "afuera" un lugar colectivo de transgresión simbólica —a través de las máscaras con que se cubren e identifican 
Volteemos la mirada ahora a los proyectos de nación que nuestro país ha instrumentado a lo largo de las diferentes vidas de su sistema político, parte como elemento unificador de los discursos, y parte como elemento constructor de un imaginario social de progreso y modernización, y estaremos en presencia de nuestra identidad nacional. Descubramos ahora los nexos que vinculan una y otra concepción, fundiéndolas a veces en una sola, y apuntaremos a destacar uno de los mecanismos modernizadores más importantes en operación: el de la construcción de los discursos de la modernidad a través de los medios de comunicación colectiva.

Esos proyectos de nación se han transformado simplemente porque hace diez años la discusión política en torno de si entrar o no al TLC se centraba sobre el tema de la soberanía. Hoy ya nadie se preocupa de la soberanía; nuestra relación con Estados Unidos y Canadá es un hecho y estamos más que preocupados porque dicha relación se lleve en buenos términos. Sabemos que con nuestras economías ligadas, hoy más que nunca es importante conseguir el aval económico y político de Estados Unidos.

Lo que sí se cuestiona es nuestra nacionalidad, nuestro amor por la patria.

Los partidos de oposición al partido en el poder le reprochan haberle dado la espalda al proyecto revolucionario y pretender modificar las estructuras legales y de la administración pública para mantener el proyecto económico. En el trasfondo, sin embargo, coexiste una identidad cultural cambiante, con una identidad nacional que se quedó anclada en un sistema político, en un régimen y en una estructura de partido que ya no corresponde a la realidad actual.

Quienes todavía apuntan hacia el problema de la identidad en cualquiera de sus dos acepciones, sea cultural o nacional, quizás se quedan cortos al señalar sólo una parte del problema; el análisis debiera centrarse en las formas en las que estructuralmente se están entrelazando los mecanismos culturales con los mecanismos de cooptación política y económica. Quizás la discusión debiera centrarse más en términos de la discusión de un proyecto nacional, que aglutina en sus propuestas la premisa inherente de una fusión cultural característica de una modernización esencialmente económica; y claro, frente a las protestas de algunos sectores intelectuales ante las propuestas de ese proyecto modernizador, en la manifestación clara, una vez más, de una preocupación no tanto cultural sino política, relativa a la ausencia de participación social en la definición de un proyecto modernizador nacional.

Si revisamos el proceso de creación de identidad nacional por el que atraviesa nuestro país, y la participación del Estado contemporáneo en la creación de semejante identidad, podemos establecer cinco etapas fundamentales, según Carlos Monsiváis: en la primera (1910-1920), los cambios que se dan no significan el fin de un sistema económico, pero denuncian las presiones de una revolución social y cultural en torno al nuevo trato del individuo con 
puesta interesante" que trata de hacer algo por los que son iguales a ellas. La banda es el anclaje principal para la propuesta simbólica de "hacer algo por la banda, por los jóvenes, por la sociedad".

En ese sentido, sabemos que la banda es una forma espontánea y natural de agregación juvenil entre los sectores populares y de clase media baja urbanos. Es una forma de organización paralela o alternativa a las tradicionales y solidaria entre pares que pertenecen a las mismas generaciones, a la vez delimitadora - defensiva-y, ocasionalmente, impugnadora. La participación en estas miniredes grupales constituye la primera marca de su delimitación como jóvenes frente a los adultos.

En el caso de las CHAP, la banda constituye el espacio simbólico donde chavos y chavas son co-partícipes solidarios en la vida de cada uno durante algún tiempo. Pero, mientras que en la banda intragenérica es reconocida como espacio de transgresión simbólica y, también, de reproducción de desigualdad e inequidad entre sexos.

\section{Identidad: distancia de rol y nuevas construcciones}

De acuerdo con Jean Claude Kaufmann y siguiendo nuestro eje microscópico, el estudio de la identidad obliga a introducir la discusión sobre la articulación roles-identidad. Para ese autor, el rol es un elemento decisivo para la redefinición de la identidad, la cual supone un pasado incorporado, reservas acumuladas, esquemas interiores que se reactivan y participan en ésta. Lo anterior supone una concepción dinámica de la identidad que se reestructura por la interacción y la socialización de un momento en particular. Kaufmann señala que "al involucrarse en ciertos roles y al negociar los términos de ajuste, los actores participan en la recomposición de su patrimonio de esquemas incorporados y redefinen los cuadros de su identidad". Al parecer, ésta se forja a partir de una sucesión de roles, o bien, a la manera de Mead, “el individuo no está más que en los roles sucesivos que ocupa".

Pese a ello, al interior del encuadre social el individuo tiene la capacidad de distanciarse del rol. Kaufmann anota que la ocupación o el desempeño de un nuevo rol produce inevitablemente un desplazamiento de la construcción identitaria, hay un pequeño conflicto entre la antigua y la nueva concepción del yo. Con frecuencia el antiguo rol se resiste o se esconde temporalmente listo a reaparecer. En suma, la identidad se conforma en una sucesión de roles que conectan el pasado con el presente, reactualizándose de acuerdo con los momentos particulares de socialización. 
que es la cultura. En este último caso la cultura actúa como elemento de adhesión e identificación de la identidad, pero sólo en la medida en que su variedad sea respetada y pueda manifestarse libremente (Fuentes, 1991a, 15).

Fuentes (1992: 29) apunta que la modernidad ha sido nuestro fantasma constante, nos ha acompañado a lo largo de toda nuestra historia. La realidad, dice el autor, es que tenemos que hacer cuentas con nuestro pasado para enfrentarnos a esa historia y asumir lo que somos. Mientras no asumamos lo que somos no podremos seguir adelante. Ahora bien, parte del reconocimiento de la propia identidad estriba en el reconocimiento del otro, en la conciencia de lo extraño, que acaba por enriquecernos.

La problemática de la apropiación intercultural en nuestro tiempo, radica en la aceleración de los procesos que resultan de la nueva tecnología de información, y particularmente en un momento como el actual, en que las identidades son políglotas, multiétnicas, migrantes y hechas con elementos de varias culturas, cuando por primera vez (en este nuevo siglo), la mayor parte de los bienes y mensajes que recibimos no son producidos en el propio territorio, ni llevan vínculos que los liguen a la comunidad nacional, sino que más bien pertenecen a un sistema desterritorializado (García Canclini, 1992: 32-33).

Ahora bien, en una situación de interculturalidad como ésta, las culturas se configuran no sólo por las diferencias entre culturas desarrolladas de manera desigual, sino por las maneras desiguales en que los grupos se apropian de elementos de varias sociedades, los combinan y los transforman. El objeto de estudio de una situación tan particular como la que ahora se nos presenta, dice García Canclini (1992: 34), debe ser no sólo la diferencia, sino también la hibridación.

Otro antropólogo, Guillermo Bonfil Batalla, en uno de sus últimos trabajos, apuntaba directamente sobre las consecuencias culturales del Tratado de Libre Comercio para nuestro país; en dicha intervención decía:

En el terreno cultural, en efecto, una de las posibilidades más sugerentes y positivas del TLC sería la de facilitar la circulación más libre de ideas y de los valores alternativos que éstas conllevan. Por decirlo en términos telecistas: una oferta mayor y más divertida de ideas y valores capaces de dar sentido a nuestra existencia [...] El problema no está en el TLC [...] sino en los mecanismos sociales y políticos que seamos capaces de instrumentar en la sociedad mexicana para asegurar el mejor uso posible de esa prerrogativa en beneficio auténtico de nuestra propia sociedad plural. ${ }^{17}$

17. Bonfil Batalla, Guillermo (1991), "Dimensiones culturales del Tratado de Libre Comercio”, en México Indigena, núm. 24, septiembre, p. 10. 
Respecto de la relación con los padres, novios, chavos, bandosos y machines, ellos generalmente aparecen como no comprometidos, débiles, irresponsables y pasivos en oposición al "nosotras como chavas banda: las que hacemos algo", o bien, las chavas banda como "luchadoras sociales".

Aunque las CHAP distinguen a los chavos banda propositivos (sanos, políticos, "limpios" o no adictos) del resto, se percatan de que "hay muy pocos chavos que son conscientes y que apoyan la situación de las chavas".

\section{Conclusiones}

Los relatos parecen mostrar una confrontación entre dos escenarios: por un lado, el inexorable destino de su clase social de pertenencia (cuidar hijos propios y ajenos, trabajar de sirvienta y esperar, en el mejor de los casos, a que alguno de los chavos - golpeador o borracho- de su mismo sector social la haga su esposa); por el contrario, la opción de la calle y la banda como lugar imaginario que le permite organizarse, tener vida propia y además ir asumiendo valores, modelos y maneras de comportamiento distintos a su universo cultural de origen. Su salida del ámbito familiar doméstico, el reto y la búsqueda por ser diferentes es el motor que las impulsa para adoptar como propios los valores de la pequeña burguesía intelectual: espíritu crítico, búsqueda de un "mundo mejor", cuestionamiento de la vida social.

Así, en las CHAP se enfrentan dos principios organizadores de la vida cotidiana, de reconocimiento, de interpretación, de significación, dos ethos: por un lado, su inevitable destino social y, en contraparte, la propuesta cultural y bandosa que les permite otear nuevos y prometedores horizontes, participar en propuestas culturales alternativas, ser miembro de movimientos impugnadores, ser defensora y representar a las mujeres; en resumen, ser una luchadora social y, con ello, reconocida y exitosa.

Tal vez en posteriores prospecciones podamos, a manera de Lavive, explorar el "mundo de la vida" de las CHAP desde una idea aparentemente simple; esto es, la vida de los hombres y las mujeres se inserta en un espacio tridimensional: la inserción en el tiempo, en el espacio y en lo social. Quizá desde esta propuesta podremos profundizar en el reconocimiento de la confluencia confrontación o contraste de estos dos principios organizadores o ethos de las vidas de nuestras protagonistas. 


\section{Bibliografía}

Cornejo, Inés y Maritza Urteaga (1998): "Movimiento punk e identidad femenina”. Revista Chasqui, número 62, Ecuador.

Lalive D'penay, Christian: Relato de vida, ethos y comportamiento: por una exégesis sociológica. Universidad de Ginebra, s.e., s.f.

Kaufmann, Jean Claude: Rôles et identité: L'exemple de l'entrèe en couple, s.e., s.i., s.f.

Ritzer, George (1997): Teoría sociológica contemporánea. McGraw Hill, España.

Taylor, S.J. y Bodgan, R. (1992): Introducción a los métodos cualitativos de investigación. Paidós, Argentina.

Urteaga, Maritza (1995): Nuevas culturas populares. Rock mexicano e identidad juvenil en los 80's. Tesis de maestría en Antropología Social. Escuela Nacional de Antropología e Historia (ENAH), México.

Urteaga, Maritza e Inés Cornejo (1996): Cultura e identidad femenina: el caso de las Chavas Activas Punk's", en Espacios de Comunicación, número 1. Serie Azul, División de Ciencias del Hombre, Departamento de Comunicación. Universidad Iberoamericana, México. 